\title{
Auge y Crisis de la Industria Azucarera Venezolana*
}

\author{
Catalina Banko** \\ Karelys Abarca***
}

\begin{abstract}
This article examines the features of the sugar industry and its development in Venezuela from the $19^{\text {th }}$ Century until the present situation. In the 1930s the domestic demand of sugar had increased faster than the national production due to the inadequate technology and outdated crop's practices. This situation changed in the 1950s with the new government economic policies. The expansion of the Venezuelan sugar's sector was guided by the government strategy to raise levels production through large public investments to improve productivityenhancing technologies, and loan provision for private mills. Between the 1970s and the 1980s, this industry began to undergo adverse conditions in the context of macroeconomic unbalances. Venezuelan sugar's sector currently faces a deep crisis rooted in many structural factors: low productivity, inefficient technology, undercapitalization of growers and mills and inappropriate agricultural policies.
\end{abstract}

Keywords: Mills, Sugarcane Farms, Venezuela, Expansion, Crisis

Resumen: En el artículo se analizan las características y la evolución de la industria azucarera en Venezuela desde el siglo XIX hasta la actualidad. En los años treinta, la demanda interna fue creciendo a un ritmo más acelerado que la producción nacional a causa del empleo de técnicas inadecuadas y la aplicación de atrasados métodos de cultivo. Esta situación se modificó en los cincuenta a través de la implementación de nuevas políticas económicas. La expansión de la industria azucarera venezolana estuvo orientada por la estrategia gubernamental destinada a incrementar la producción mediante grandes inversiones para mejorar la productividad y el adelanto tecnológico y el suministro de créditos al sector privado. Entre los años 70 y 80, el sector comenzó a sufrir condiciones adversas en el contexto de graves desequilibrios macroeconómicos. La industria azucarera venezolana confronta actualmente una profunda crisis provocada por múltiples factores de carácter estructural: baja productividad, tecnología ineficiente, escasez de capitales para la inversión en haciendas y factorías y la aplicación de políticas agrícolas inapropiadas.

Palabras-clave: Centrales Azucareros, Haciendas, Venezuela, Auge, Crisis

\footnotetext{
Este trabajo forma parte de una investigación más amplia sobre la historia del azúcar en Venezuela, que cuenta con el auspicio del Fonacit-Bid y el Consejo de Desarrollo Científico y Humanístico de la Universidad Central de Venezuela, organismos a los cuales expresamos nuestro agradecimiento por el apoyo brindado.

" Catalina Banko, Profesora en la Escuela de Sociología y en la Escuela de Economía de la Universidad Central de Venezuela.E-mail: catabanm801@cantv.net

Profesora en la Escuela de Sociología y en la Escuela de Economía de la Universidad Central de Venezuela. Email:karelysaba@cantv.net
} 


\section{Introducción}

La insuficiencia de la producción agrícola e industrial para satisfacer la demanda interna, a pesar de los múltiples proyectos de fomento presentados en el transcurso de las últimas décadas, es uno de los graves problemas que actualmente confronta la economía venezolana. El escaso desarrollo de la agricultura ha motivado el gran incremento de las importaciones, situación que se refleja claramente en el caso del azúcar, artículo esencial en la alimentación cotidiana e insumo para numerosos rubros industriales, además de constituir una actividad que tiene alta capacidad generadora de empleo. Por ello, consideramos que el análisis del proceso de desarrollo de la industria azucarera venezolana, en sus etapas de auge y crisis, constituye un tema de interés, que puede contribuir a la comprensión de la dinámica de este importante sector de la agroindustria nacional.

\section{La explotación azucarera tradicional}

Durante el siglo XIX la economía venezolana permaneció sustentada en la producción de café y cacao para la exportación. Debido a la especificidad de este género de explotación agrícola, no se generaban encadenamientos productivos que permitieran difundir efectos dinamizadores en el conjunto de la economía y, al mismo tiempo, tampoco fue posible, aumentar considerablemente la producción debido a la limitada demanda mundial. Profundas deficiencias estructurales obstaculizaban el desarrollo de la agricultura y del sector manufacturero, entre las cuales sobresalían: la segmentación de los mercados, la escasa circulación monetaria, la limitada inversión de capitales, las dificultades del transporte y el atraso técnico, a lo que se agregaba la ausencia de un sector capitalista sólido, la inestabilidad política y las recurrentes crisis económicas.

En medio de este panorama desalentador se desenvolvió la producción azucarera que llegó a tener gran extensión en el territorio venezolano desde la etapa colonial. Sin embargo, dicha actividad tropezó con sinnúmero de dificultades que impidieron su desarrollo posterior, de manera semejante a lo que estaba ocurriendo con el conjunto de la agricultura venezolana.

Por entonces, la explotación azucarera se llevaba a cabo en la "hacienda-trapiche", unidad productiva en la cual estaba integrado el proceso completo desde el cultivo de la caña hasta la elaboración de papelón y aguardiente, mediante el empleo de fuerza animal e hidráulica. Durante el siglo XIX se continuó utilizando el mismo sistema de producción de las tradicionales haciendas coloniales, aunque se introdujeron nuevas variedades de caña y se incorporaron lentamente algunas innovaciones técnicas, como la utilización de máquinas a vapor.

Junto al sistema predominante de la tradicional "hacienda-trapiche", fueron tomando cuerpo diversas iniciativas para organizar unidades productivas más complejas, con la 
incorporación de avances técnicos y, de manera especial, mediante la explotación intensiva de la mano de obra esclava, hecho que se materializó casi exclusivamente en el Litoral Central. Entre los años treinta y cuarenta del siglo XIX, adquirieron gran importancia varias haciendas azucareras con siembras abundantes, gran número de esclavos e inversiones de capital relativamente considerables, lo cual se configuró en un importante intento por modernizar la producción, con el objetivo de exportar hacia los Estados Unidos. En una de estas haciendas, por ejemplo, se llegaron a emplear hasta 230 esclavos, lo cual constituía un caso muy llamativo en la época, debido a la escasez de ese tipo de mano de obra (TROCONIS, 1979, p. 84-85).

Sin embargo, la escasa capacidad de competencia frente a los grandes exportadores del Caribe, determinó el posterior fracaso de estos primeros proyectos modernizadores de la explotación azucarera. Al respecto, es importante señalar que desde el período colonial solamente algunas islas del Caribe habían adquirido carácter dominante en las exportaciones de azúcar. Este no era el caso de Venezuela, donde las haciendas de caña, en su mayor parte, no estaban adaptadas para producir grandes volúmenes ni para la obtención de elevados niveles de productividad, sino exclusivamente para abastecer pequeños mercados locales (RODRÍGUEZ, 1986, p. 45-66).

En las últimas décadas del siglo XIX, se comenzó a intensificar la preocupación por aumentar el rendimiento de la caña. Este incremento podría lograrse con la aplicación de nuevos procedimientos utilizados por los "ingenios centrales", sistema que implicaba la separación de las tareas agrícolas y las de fabricación del azúcar. Mayores rendimientos podrían obtenerse, entonces, mediante una "explotación racional" y la reorganización de las haciendas incorporando mejoras en los cultivos, y formar así agrupaciones de haciendas que beneficien sus cañas en "oficinas centrales". De esta manera, podría llegar a exportarse el producto con los consiguientes efectos positivos para la economía nacional (EL ECONOMISTA, 1890).

En el año 1890, Federico Vollmer, propietario de la conocida hacienda El Palmar ubicada en Aragua, tras una experiencia de diez años en los ingenios cubanos, dio a conocer un proyecto para la creación de centrales, con el propósito de modernizar la elaboración de azúcar y promover las exportaciones de este producto. Dichas propuestas no prosperaron en aquellos años por el temor de los hacendados a que el incremento de la producción pudiera ocasionar una baja de los precios, ya que las perspectivas para las exportaciones eran bastante inciertas, en circunstancias en que se estaban extendiendo de manera vertiginosa los cultivos de remolacha azucarera en Europa (EL ECONOMISTA, 1890).

A lo largo del siglo XIX, el estancamiento de la actividad azucarera estaba inscrito dentro de la crisis general de la producción agrícola sustentada en la exportación de café y cacao. El ambiente económico no era favorable para la expansión de la agricultura, 
debido a la incidencia de factores, tales como, la escasez de capitales, el reducido tamaño del mercado interno, la falta de mano de obra y las dificultades para la comunicación interregional, a lo que se unían los problemas derivados de la inestabilidad política y la consiguiente falta de confianza para la inversión.

\section{Fundación de los primeros centrales azucareros}

A inicios del siglo XX se intensificó el interés por instalar ingenios centrales, en un ambiente más propicio para la inversión de capitales en nuevas empresas económicas. La fabricación de papelón y azúcar era una importante actividad en aquel entonces. Entre 1910 y 1911 , el capital perteneciente a los cultivadores de caña representaba el 24\% del total de las inversiones en el sector agrícola. Esta elevada proporción respondía a la peculiaridad de la hacienda-trapiche, la cual requería abundante mano de obra y considerables erogaciones para la adquisición de herramientas y máquinas (FOMENTO, 1913).

En el contexto de la gran expansión de las industrias a nivel mundial comenzaron a alimentarse en Venezuela grandes expectativas con relación a la posibilidad de producir azúcar, especialmente para la exportación, mediante el sistema de centrales. La primera empresa de esta naturaleza fue la C.A. Central Azucarero, constituida en el Zulia en 1912. También en dicho estado, se estableció al año siguiente la firma Venezuela Sugar Company, que en 1920 pasó a denominarse Central Venezuela. El Central Tacarigua (Carabobo) fue fundado en 1913, siendo uno de sus principales accionistas el general Juan Vicente Gómez. En el mismo año se instaló el Central La Ceiba (Trujillo), cuya producción podía ser transportada por el Lago de Maracaibo con la consiguiente reducción de los fletes. Estas primeras industrias, a excepción del Tacarigua, se localizaron en la región próxima al Lago de Maracaibo, ubicación que brindaba una serie de ventajas por las facilidades de transporte y la fertilidad de las tierras circundantes. El principal objetivo de estos centrales residía en la producción de azúcar para la exportación, debido a que el mercado interno era todavía muy limitado. En aquel tiempo eran escasas las posibilidades de los productores para competir en el mercado externo a causa de los bajos rendimientos de la caña y las primitivas técnicas aplicadas al procesamiento de la misma (BANKO, 2003).

En los años de la Primera Guerra Mundial, la demanda de azúcar se elevó notablemente a consecuencia de la interrupción de los cultivos de remolacha en Europa. Al concluir la contienda esa tendencia se acentuó aún más, al punto que en 1919 las exportaciones procedentes del Central Venezuela, ubicado en el Zulia, se duplicaron con respecto al año anterior, incentivando así la expansión de los cultivos de caña (Ministerio Hacienda, 1908-1922).

El auge de la primera posguerra fue relativamente breve, ya que a mediados de 1920 comenzaron a emerger los signos de una honda crisis, que se agravó al año siguiente, como consecuencia de las perturbaciones derivadas del proceso de transición hacia la paz europea. 
Los mercados, que aún no habían llegado a recuperarse de los efectos del conflicto, se saturaron rápidamente, por lo cual disminuyó la demanda y los negocios sufrieron una repentina paralización que ocasionó la caída de los precios de las materias primas. Más graves aún fueron los efectos de la crisis mundial de 1929 que significaron un duro golpe para la industria azucarera, ante la caída vertical de los precios en el mercado internacional. En tales circunstancias, las exportaciones de azúcar prácticamente desaparecieron del cuadro del comercio exterior venezolano (Ministerio Hacienda, 1919-1930).

Con relación a esta primera etapa de los centrales azucareros, debemos resaltar que su creación estuvo vinculada con las posibilidades que brindaba el mercado externo en la segunda década del siglo. Por un lado, la fertilidad de las tierras zulianas posibilitaba un alto rendimiento de la caña de azúcar y, por otro, la ventajosa localización a orillas del Lago de Maracaibo permitía abaratar el transporte. Estas condiciones favorecían la producción de azúcar con destino a las exportaciones, lo cual se constituyó en el principal objetivo de los primeros centrales en Venezuela. La coyuntura de la Primera Guerra Mundial y la ampliación de la demanda entre 1919 y 1920 contribuyeron al acelerado crecimiento del sector azucarero. Precisamente, los años de mayor auge del Central Venezuela coincidieron con el gran incremento de las exportaciones. Estas circunstancias explican el interés demostrado por algunos representantes del sector mercantil zuliano por invertir en la instalación del mencionado central. Sin embargo, el estallido de la crisis de 1929 y sus prolongados efectos en la economía mundial provocaron la caída de las exportaciones, por lo que la producción del Central Venezuela debió orientarse hacia el mercado interno.

\section{Acción estatal y expansión de la industria azucarera}

A partir de 1936 se introdujeron importantes cambios en materia económica y social que contribuyeron a configurar un nuevo perfil de la acción estatal. La creación del Banco Industrial de Venezuela (1937), aunque limitada inicialmente al financiamiento de pequeñas empresas manufactureras, tuvo significativa importancia en el proceso de modernización económica. A través del Plan Trienal (1938-1941) se esbozaron los lineamientos fundamentales y las acciones concretas de la nueva política económica y social. Se trató del primer programa económico puesto en práctica en Venezuela, además de constituir el punto de partida de la aplicación concreta de políticas sociales, principalmente en las áreas de educación y salud (Ministerio Fomento, 1939-1940).

Con base en la nueva concepción en torno al papel del Estado, el Plan Trienal ofrecía una propuesta de modernización de las actividades productivas. Se proyectó el fomento de aquellas industrias de consumo nacional que permitieran ampliar el capital y el trabajo, además de disminuir las elevadas importaciones de artículos manufacturados. 
En el marco de las nuevas políticas del Estado, el panorama industrial varió radicalmente en las décadas siguientes. La expansión de ciertos rubros manufactureros, como alimentos y bebidas, requería crecientes suministros de azúcar, al tiempo que los cambios en los hábitos de consumo de la población urbana condujeron también a una ampliación de la demanda de azúcar refinada, en detrimento del tradicional papelón. Dado que la producción nacional ya no lograba satisfacer las necesidades del mercado interno, fue necesario acudir a las importaciones. Durante la Segunda Guerra Mundial el desabastecimiento se agravó, debido a la insuficiencia de la producción y a las dificultades para su obtención en el exterior. Precisamente, en estos años se intensificó el interés por la instalación de nuevas plantas industriales. Una de estas iniciativas se materializó con la fundación del Central Matilde (Yaracuy) en 1946 (Ministerio Agricultura y Cría, Memoria, 1948).

Los datos referentes a la producción de azúcar nos revelan claramente la situación de este rubro agroindustrial. En el año 1945 se produjeron apenas $27.241 \mathrm{Tm}$ frente a un consumo que ascendía a $38.659 \mathrm{Tm}$, por lo cual era necesario acudir a las importaciones para abastecer el mercado interno. Ante la escasa capacidad de producción de los centrales existentes, la situación deficitaria se agravó, especialmente en 1948, cuando la producción disminuyó a 26.552 Tm y las importaciones se elevaron a 56.171 Tm. Posteriormente, la incorporación del Central Matilde y la modernización del Central Venezuela posibilitaron un incremento de la producción relativamente significativo: $50.369 \mathrm{Tm}$ en 1950, aunque este volumen no era todavía suficiente como para abastecer el consumo local (Ministerio Fomento, 1958).

\section{Etapa de auge de la producción azucarera}

Ante la creciente demanda interna y la insuficiente producción nacional, en los años cincuenta el gobierno decidió asumir en forma directa el impulso de la producción de azúcar, el cual se había convertido no solamente en un artículo fundamental para el consumo doméstico, sino también para el uso industrial, ya que eran muchos los rubros manufactureros que utilizaban azúcar como insumo en sus procesos productivos.

En este contexto, se pusieron en práctica diversos programas para impulsar la agricultura a través de la Corporación Venezolana de Fomento (CVF) y del Banco Agrícola y Pecuario (BAP). Como parte de esa política, fue diseñado el Plan Azucarero Nacional en 1950, cuyo objetivo fundamental consistía en la asistencia financiera y técnica a los centrales por parte del Estado, el cual se convirtió también en propietario de varios establecimientos azucareros. Con el propósito de estimular la modernización del sistema productivo, se crearon estaciones experimentales para estudiar los problemas relativos a la calidad del suelo, rendimiento, control de plagas y adelantos técnicos. La CVF intervino de manera directa en la fundación de los centrales Motatán (Trujillo), Cumanacoa (Sucre), 
Tocuyo (Lara) y Ureña (Táchira), que en 1954 ya se encontraban en funcionamiento. Asimismo, tomando en cuenta las amplias potencialidades productivas de las tierras larenses, la CVF participó en la instalación del Río Turbio (Lara) en 1956. El antiguo Central Tacarigua recibió cuantiosas inversiones que posibilitaron un gran aumento de su producción a mediados de esa década (YÉPEZ, 1970, p. 33-57).

En cuanto al sector privado, también surgieron importantes iniciativas, como las de los centrales La Pastora (Lara) en 1953 y de El Palmar (Aragua) en 1956. Al año siguiente se constituyó el Central Yaritagua (Yaracuy) con capital proveniente de los empresarios fundadores de El Palmar. Gracias a la incorporación de las nuevas factorías a la producción de azúcar, la misma se elevó a 144.000 Tm en 1955 y a 198.586 Tm al año siguiente. Se alcanzó así el completo abastecimiento interno, con lo cual las importaciones prácticamente desaparecieron, e incluso fue posible exportar parte de los excedentes (Ministerio Fomento, 1960).

Esta situación estaba expresando el grado de dinamismo que adquirió la agroindustria azucarera en ese período, en el marco de un conjunto de políticas adoptadas por el Estado. Se inició así la etapa de auge de la producción azucarera, lo cual tuvo además positivas repercusiones por la generación de empleos tanto directos como indirectos. En 1955 se creó la Distribuidora Venezolana de Azúcares, con el fin de sistematizar el sistema de distribución y ventas de azúcar y reducir así los costos y homogeneizar las condiciones del mercado para evitar la guerra de precios entre los diversos centrales.

En este contexto, varió sustancialmente el panorama de la industria azucarera a nivel nacional, ya que el eje se desplazó de la región zuliana hacia la región central y centro occidental. La producción del Central Venezuela aumentó, pero en una proporción pequeña en comparación con los nuevos centrales, tanto públicos como privados, creados a partir de 1946. Los mayores incrementos se localizaron en Lara, Yaracuy y Aragua, principalmente. En el marco de este desplazamiento de los centros productores, se redujo notablemente la importancia relativa de la región zuliana en este rubro. En el año 1945, el Central Venezuela había aportado el 38\% de la producción nacional y el Tacarigua el 17\%. La situación varió totalmente en los años posteriores. En 1963 el central zuliano producía solamente el $7 \%$ y el Tacarigua el $6 \%$ del total nacional. En el mismo año, la producción de El Palmar representaba el 20\%, Río Turbio: 17\%, Matilde: 14\%, El Tocuyo: 12\%, Yaritagua: 7\% y Cumanacoa: 7\% (ABREU, 2001, p. 319-320).

La aplicación del primer Plan Azucarero, en los años cincuenta, generó una profunda transformación de la explotación azucarera, mediante la intervención directa del Estado para desarrollar centrales con mayor potencial productivo en las regiones que eran consideradas estratégicas por sus cualidades para la explotación agrícola y su posibilidad de acceso a los mercados de consumo más importantes, tanto por la existencia de centros 
industriales como por la concentración de población. En estas circunstancias, el mercado quedó dominado por los nuevos centrales, tanto públicos como privados, que contaban con capitales de magnitud y estaban equipados con moderna tecnología.

La puesta en funcionamiento de todos estos centrales en la década de los cincuenta, trajo consigo un gran crecimiento de la producción que provocó entre 1956 y 1957 un excedente considerable que, al no ser absorbido por la demanda interna, fue exportado a precios por debajo de los costos de producción, provocando pérdidas cuantiosas. Esta situación ocasionó la posterior caída de la producción por debajo de la demanda, provocando el incremento de las importaciones en 1958.

Debido al crecimiento de la producción que tuvo lugar en los años cincuenta, bajo la meta de garantizar que no se produjeran excedentes en la oferta de azúcar en el mercado nacional y evitar el deterioro de los precios, se adoptó como solución la congelación de los precios de venta, lo que terminaría convirtiéndose en la medida que golpearía con mayor dureza a este sector industrial (YÉPEZ, 1970, p. 53-59).

La expansión de la industria azucarera prosiguió durante los años sesenta, en el contexto de la aplicación del modelo de Sustitución de Importaciones, a través del suministro de créditos al sector y a la creación en general de políticas proteccionistas, como parte de una concepción en que el Estado debía actuar como "promotor" del desarrollo económico. En estos años se registró un entorno macroeconómico estable, con una baja inflación expresada en el incremento del Producto Interno Bruto (PIB) a precios constantes, así como un tipo de cambio con una tasa de depreciación baja. La tasa de interés activa creció a un ritmo promedio de 5,23\% anual y la inflación de costos en el sector agrícola fue poco significativa, apenas del $2 \%$ anual (BCV, 1991).

En la primera etapa de aplicación del modelo de Sustitución de Importaciones los resultados fueron exitosos, ya que entre 1962 y 1965 se registró una tasa media interanual de 10,2\% en el crecimiento del Producto Industrial Bruto, aunque luego disminuyó a $4,7 \%$ entre 1965 y 1969 , reflejando pérdida de dinamismo del desarrollo industrial. Es necesario destacar que este proceso industrializador se caracterizó por una elevada dependencia de la importación de insumos y bienes de capital, lo cual determinó una tendencia declinante en la competitividad a través de la inflación de costos que se transfirió del sector manufacturero a todas las demás actividades (BCV, 1991).

La industria azucarera de los años sesenta mostró una evolución positiva en casi todas las variables: se alcanzó el autoabastecimiento en el mercado interno de consumo e incluso tuvo lugar un significativo aumento de las exportaciones a lo largo de la década. Asimismo, se crearon organismos especializados en la modernización de los sistemas de producción y la elevación de la productividad. 
El IV Plan de la Nación, puesto en práctica en 1970, estaba inscrito en el mismo esquema de sustitución de importaciones diseñado a inicios de los sesenta. Con relación al sector agrícola, se destacó su importancia en el sistema productivo nacional y en la integración interna del mismo, así como en su capacidad de generación de empleo (CORDIPLAN, 1970). También acentuó la relevancia de la producción de caña de azúcar en algunas regiones del país y la presencia de ese sector en el mercado internacional. La estrategia para el desarrollo agrícola se orientó al aumento de la producción y al uso eficiente de los factores productivos .

El resultado de las políticas destinadas a promover la sustitución de importaciones se expresó en un acelerado aumento de la producción azucarera, que se elevó de 214.616 Tm en 1961 a 493.354 Tm en 1972. En este último año, se registró incluso el más alto nivel de las exportaciones venezolanas de ese artículo: $152.338 \mathrm{Tm}$. La producción de caña de azúcar alcanzó su punto máximo en el año 1967 (4.345.426 Tm.) con una alta tasa de crecimiento anual de 8,95\% (Ministerio Fomento, 1974).

Los resultados obtenidos fueron el producto de un gran esfuerzo financiero, técnico y gerencial, concertado entre los sectores público y privado, los centrales, cañicultores, patrones, obreros y técnicos de fábrica y campo. Además, se ampliaron las áreas sembradas de caña y se dio un uso más racional a los recursos disponibles.

\section{Crisis de la agroindustria azucarera}

La industria azucarera ha constituido una de las actividades de mayor relevancia dentro del desarrollo agroindustrial venezolano, no sólo por su capacidad para generar empleo directo e indirecto a nivel regional, sino también para incrementar el grado de encadenamiento productivo en la economía nacional. A pesar de que en el período 1961- 1973 el volumen de producción creció a una tasa de 6,2\% anual, a partir de 1973 la industria de refinación y la agroindustria en general, manifestaron síntomas de desaceleración en la producción, pérdida de dinamismo en su aporte al PIB y a la producción manufacturera y una situación financiera riesgosa (Ministerio Agricultura y Cría, Anuario, 1974-1979). Esta situación obedeció a la carencia de una política azucarera coherente que resolviese el problema de abastecimiento de las materias primas, comercialización del producto, incremento de la productividad en el campo, incentivo a

\footnotetext{
${ }^{1}$ En los setenta aumentó más la inflación en el sector agrícola (11,26\% de crecimiento promedio anual) que la inflación en el sector industrial (8,20\% de crecimiento promedio anual) y sector azucarero (7,13\% de crecimiento promedio anual); este resultado fue una consecuencia del aumento de los costos provocados por la aplicación de las políticas del plan.
} 
la investigación y desarrollo en campo y fábrica y, principalmente, una política de reestructuración de los precios (tradicionalmente regulados por el Estado), suficientemente planificada, con el fin de proteger al consumidor, pero sin afectar al productor a nivel de costos. Además, la ausencia de una política financiera eficiente (menores requerimientos burocráticos e intereses bajos), al servicio del cañicultor, contribuyó a que el sector ingresara en una prolongada fase de crisis en su ciclo productivo.

El boom petrolero de 1973 significó para Venezuela un incremento extraordinario de los ingresos fiscales. En 1975 se inició la aplicación del V Plan de la Nación, que proyectaba el desarrollo del aparato productivo interno por medio de grandes inversiones públicas. El Estado se convirtió así en el resorte fundamental de la economía nacional, siendo la orientación del gasto público interno uno de los factores determinantes en el proceso de asignación de recursos en la economía. Con el fin de materializar esos ambiciosos planes, el Estado, rector de la economía y empresario a la vez, debió acudir al financiamiento externo. Sin embargo, cuando los precios petroleros comenzaron a descender, emergieron las limitaciones estructurales del modelo, ya que no era posible sostener planes de inversión con estancamiento del ingreso petrolero y creciente déficit en la balanza de pagos. Con el V Plan de la Nación se intentó profundizar el proceso de sustitución de importaciones, avanzando en la etapa de sustitución de bienes intermedios y de capital, aunque se omitió la necesidad de fomentar las exportaciones industriales competitivas internacionalmente (CORDIPLAN, 1975).

En ese contexto, se fue profundizando la dependencia del sector privado de la tecnología importada, de la protección del Estado y del ingreso petrolero. Durante los años setenta, el sector industrial no llegó a fortalecer ni desarrollar los elementos determinantes de la competitividad internacional ${ }^{2}$, y siguió creciendo bajo el amparo del Estado y el esquema de tipo de cambio apreciado (4,30 Bs./US\$).

Como consecuencia de esa situación, la industria azucarera sufrió una grave crisis con una drástica caída de la producción y de los indicadores de rendimiento. La apreciación del tipo de cambio en los primeros años de la década de los setenta, provocó la disminu-

\footnotetext{
${ }^{2}$ Michael Porter manifiesta en el libro La ventaja competitiva de las Naciones los determinantes de la competitividad internacional, a saber: la estructura y estrategia de la rivalidad de las empresas, las condiciones de la demanda interna (estructura de la demanda, existencia de compradores entendidos y exigentes, tamaño de la demanda interior, internacionalización de la demanda interior), existencia de sectores conexos y de apoyo (aspecto involucrado al grado de integración interna de una actividad a la economía nacional) y las condiciones de los factores productivos (dotación de recursos humanos, físicos, conocimientos, capital, infraestructura y capacidad para crear factores productivos de manera constante).
} 
ción del crecimiento anual de la tasa de interés activa, fenómeno que incentivó la expansión de la demanda de créditos y el incremento de la inversión privada. Sin embargo, el auge de la industria petrolera trajo consigo el abandono de la agricultura y el estancamiento progresivo de las principales ramas agroindustriales en Venezuela, incrementando la dependencia a las importaciones de bienes intermedios, finales y de capital.

Si estudiamos con detalle el período 1973-1978, notaremos que se incrementó considerablemente la inflación en la economía, a tal punto que se amplió la brecha entre el PIB a precios corrientes y el PIB a precios constantes (una tasa de crecimiento anual de $18,20 \%$ para el primero y apenas de 5,93\% para el segundo). En materia agrícola, el V Plan pretendía llevar a cabo una política agropecuaria sustitutiva para conducir a este sector a la eficiencia y rentabilidad, así como a la modernización. No obstante, la aplicación de los lineamientos de dicho plan desembocó en un extraordinario endeudamiento externo para sostener los grandes proyectos de inversión del Estado (BCV, 1991).

Los indicadores de la industria manufacturera en el período 1971-1978 revelan que hubo una expansión significativa del empleo, un incremento del valor de la producción por establecimiento a una tasa de $4,20 \%$; un crecimiento de la densidad de capital a una tasa de 15,6\% anual, mientras la productividad media real creció levemente, aumentó la remuneración al factor trabajo y se redujo el grado de integración y elaboración interna, como muestra de la escasa articulación de los sectores productivos del país. Por otra parte, se incrementó el indicador materias primas importadas sobre materias primas totales, reafirmando la existencia de la dependencia de las importaciones en el período.

Dentro del V Plan de la Nación se inscribió el II Plan Azucarero Nacional (19751980). Sus objetivos consistían en el incremento de la producción nacional de azúcar para el pleno abastecimiento interno; el aumento del ingreso de divisas en materia de exportación azucarera; incentivo de la rentabilidad del trabajo e inversión en el sector y el establecimiento de estrategias y políticas nacionales para el desarrollo del sector azucarero en el corto, mediano y largo plazo ${ }^{3}$.

La industria azucarera durante el período de la "bonanza fiscal" reveló en sus variables un inocultable fracaso de las políticas estatales. Hubo una escasa tasa anual de

\footnotetext{
${ }^{3}$ Los objetivos específicos del plan se orientaban a: ofrecer precios accesibles; integrar la agroindustria azucarera para hacerla más eficiente a través del aprovechamiento de las economías de escala; la incorporación de planes y proyectos del sector dentro de las prioridades del plan de desarrollo de la nación y el aumento de los niveles de inversión y tecnificación con el fin de incrementar la productividad azucarera. Otro de los objetivos específicos del Plan Azucarero 1975- 1980 fue la generación de mayor empleo, mayores ingresos y desarrollo económicosocial del trabajador del sector.
} 
crecimiento en cuanto a la producción de caña de azúcar, con un valor máximo de 6.000.599 Tm. en 1976, como resultado de las expectativas positivas inmediatas que generó la aparición del Plan Azucarero. En los años posteriores, cayó el volumen de exportaciones azucareras (los valores más altos se obtuvieron en 1977 y 1978: 23 Tm. y 17 Tm., respectivamente). La mayor cantidad de azúcar refinada se produjo en 1974 (507.340 Tm.), aunque la tasa anual acumulada de la década mostró una disminución de la producción azucarera de 2,92\% (Ministerio Agricultura, Anuario, 1978).

Las importaciones azucareras crecieron a una tasa promedio anual de $92 \%$, mostrando sus valores más altos en 1978 (450.713 Tm., superior a la producción de azúcar de ese año, que fue de $346.430 \mathrm{Tm}$.) y en 1979 (343.997 Tm. de azúcar crudo importado, superior al refinado nacional de $319.824 \mathrm{Tm}$ ). Estos datos nos revelan claramente la situación de crisis que estaba atravesando la industria azucarera venezolana. La desincorporación de zonas de cultivo de caña y la consecuente reducción de la molienda por parte de los centrales, fueron las principales manifestaciones negativas. Muchos de los centrales debieron dedicarse en aquellos años críticos a refinar casi exclusivamente azúcar crudo importado (Ministerio Agricultura, Anuario, 1982).

Debido al alza de los ingresos petroleros entre 1980 y 1981 , se incrementaron las importaciones destinadas a las manufacturas e industrias básicas, acelerándose aún más el proceso de endeudamiento externo, al mismo tiempo que se producía una alarmante fuga de capitales y el descenso de las reservas internacionales. En 1982 se originó una nueva caída de los precios petroleros. Al año siguiente, el déficit de la balanza de pagos era tan alarmante y la liquidación de reservas tan grave, que provocó el cierre del mercado cambiario y el establecimiento de un régimen de cambios diferenciales; la situación se agravó con los vencimientos de deuda externa a corto plazo. Esta situación afectó de manera profunda al sector industrial, que comenzó a presentar signos de paralización. El desenvolvimiento de la economía, entre 1983 y 1988, se caracterizó por una galopante inflación de costos en los productos industriales .

La profunda caída del stock de reservas del Banco Central (BCV) en 1988 provocó una devaluación del orden del 89\%. El comportamiento del mercado cambiario presionó el aumento de la tasa de interés activa, provocando una caída en la demanda de créditos para la producción y, por lo tanto, de la inversión privada. El sistema económico se hallaba en 1988 sumido en estanflación, caída del ingreso petrolero y elevado déficit de cuenta capital en la Balanza de Pagos.

\footnotetext{
${ }^{4}$ En: Karelys Abarca y E. Mayorca: Evolución de las formas de acumulación de capital en la industria venezolana, 2001.
} 
La inestabilidad macroeconómica de los años ochenta afectó más profundamente a la industria azucarera a nivel de inflación de costos que al resto de las actividades agrícolas e industriales, pues el índice de precios del productor o IPP del sector azucarero en la década creció a una tasa anual de 11,35\%, mientras el IPP del sector industrial creció a una tasa de $6 \%$ y el del sector agrícola a menos del 1\%. Esto provocó una pérdida de competitividad internacional del azúcar, al punto que sus exportaciones comenzaron a ser irrelevantes. La producción de caña creció a una tasa promedio anual de 4,58\%, mientras que el volumen de azúcar refinado creció a una tasa de 5,37\% anual, con un pico de 556.325 Tm. en 1987. No obstante, otros indicadores asociados a la industria del azúcar reflejaban resultados menos desalentadores en el período 1987-1989: una tasa de crecimiento de la productividad real del trabajo de $20 \%$ en estos tres años; un crecimiento de $18 \%$ anual en la densidad de capital y una caída del salario relativo o remuneración del empleo de $2 \%$ anual (BCV, 1991).

El alza progresiva de los precios de insumos y materiales importados usados en el proceso de fabricación del azúcar (materias primas básicas, maquinarias y repuestos, implementos agrícolas y otros) se dispararon a niveles de hasta $300 \%$, sumiendo a la agroindustria en un profundo estancamiento debido a una estructura de costos de corto plazo ineficiente, mientras los precios de venta continuaban regulados (BÁEZ, 1981, p. 20-27).

Por otra parte, el incremento de los precios de fertilizantes, insecticidas, herbicidas, maquinaria y costo de la mano de obra, frente a un precio del producto "viscoso" o invariable, trajo como consecuencia económica natural una caída de la productividad y la rentabilidad tanto en fábrica como en campo. Todo esto propició una situación en la que el sector no era capaz de generar recursos suficientes para la inversión, ni estímulo para nuevas inversiones (ABARCA, 2003). Además, el cañicultor ante el aumento creciente de los costos y la disminución permanente de sus ingresos, ha mostrado a partir de entonces una tendencia general al abandono del cultivo, para dedicarse a la explotación de otros rubros o la realización de otras actividades, lo que se ha convertido en la causa principal que condujo a la caída de la industria azucarera en términos productivos y a la existencia de altos niveles de capacidad instalada ociosa.

\section{Amarga experiencia en el período 1989-2003}

Durante las últimas décadas, la industria azucarera ha vivido una amarga experiencia. Se despilfarraron millones de bolívares en créditos, la producción cayó de manera alarmante, no hubo esfuerzos de investigación y desarrollo de la infraestructura de producción, mientras gran número de cañicultores abandonaba el gremio. Los gobiernos continuaban empeñados en controlar los precios para proteger el consumidor, lo cual 
generó la caída de la rentabilidad y un gran déficit productivo, que hizo al mercado nacional de azúcar dependiente de un nivel importante de importaciones.

En 1989 se puso en práctica un cambio diametralmente opuesto en la dirección de la política económica, cuando el gobierno de Carlos Andrés Pérez adoptó el modelo de ajuste estructural que sugería el Fondo Monetario Internacional (FMI) para recuperar el nivel de reservas internacionales y mejorar las cuentas de la Balanza de Pagos. Los objetivos de mediano y largo plazo del ajuste estructural quedaron expresados en el VIII Plan de la Nación. La nueva política económica planteaba la necesidad de disminuir el papel del Estado en la economía por medio de la liberación de precios y de las tasas de interés, y la flexibilidad cambiaria del bolívar. Asimismo, el gobierno se propuso disminuir el déficit fiscal por medio de la reducción del gasto público, las privatizaciones y la eliminación de subsidios y protecciones arancelarias (CORDIPLAN, 1980).

A partir de 1989, el Estado inició el proceso de privatizaciones de las empresas que habían sido administradas por la CVF- Centrales Azucareros (CENAZÚCAR), para cumplir con los objetivos propuestos en el VIII Plan de la Nación. Pasaron a manos privadas, los centrales El Tocuyo, Río Turbio, Río Yaracuy (actualmente llamado Santa Clara), Portuguesa (que anteriormente había sido de capital mixto), Cumanacoa, Ureña, Santa María (que fue inmediatamente quebrado por sus nuevos dueños), Carora (anteriormente de capital mixto y que después de la privatización llegó a estar en el umbral del cierre), Río Guanare y las Majaguas, hoy llamado Santa Elena (FIV, 1994, p. 9-12).

La década de los noventa constituyó una etapa crítica en el ciclo macroeconómico de Venezuela. La amplísima brecha existente entre el PIB a precios corrientes y el PIB a precios constantes (el primero creció a una tasa de 59\% anual y el segundo apenas a $1,40 \%$ ) es un indicador del impacto inflacionario generado por la alta depreciación del tipo de cambio, que se profundizó después de la crisis financiera de 1994. Dicha crisis agravó los problemas económicos del país y se reflejó en el IPP del sector agrícola, industrial y azucarero, a través de un crecimiento de los precios a tasas cercanas al $30 \%$ anual. La altísima inestabilidad del sistema llevó al gobierno nacional en 1996 a acudir al FMI en búsqueda de financiamiento a corto plazo, por lo que debió adoptarse nuevamente un programa de ajuste estructural que formó parte de la denominada "Agenda Venezuela”. Durante esta etapa se intentó aplicar políticas sectoriales de modernización y aumento de la competitividad, con el fin de insertar a la agroindustria en el comercio internacional (BCV, 1991).

No obstante, entre 1990 y 1995, se produjo una caída de la producción de caña de azúcar a una tasa de 3\% anual (la máxima producción se alcanzó en 1992: 7.323 .498 Tm) y una disminución del azúcar refinado con destino al consumo interno. Por otro lado, en el período 1990-1994 se incrementó la productividad real del trabajo a una tasa 
anual de $6,21 \%$, como compensación de la fuerte contracción del empleo en el sector (desempleo cercano al $9 \%$ anual); un incremento de la densidad de capital del $7 \%$ anual; una disminución de la remuneración del trabajo del orden del $9 \%$ anual y una contracción de los salarios medios a una tasa anual acumulada de 3\% (ABARCA, 2003).

Actualmente, la situación de la industria azucarera y la industria en general se ha agravado con la aplicación del severo control cambiario implantado en el año 2003, que elimina las flexibilidades para la importación de crudo y otras materias primas para la refinación adicional que se requiere para cubrir el mercado interno en los períodos interzafras. El Plan Nacional de Desarrollo Económico y Social de la Nación 2001-2007 del Ministerio de Planificación y Desarrollo de la República Bolivariana de Venezuela, es de carácter profundamente intervencionista, no posee lineamientos claros en materia sectorial y no define una política específica dirigida al sector de producción de azúcar.

La política oficial azucarera aplicada actualmente en Venezuela, consiste principalmente en un conjunto de medidas que afectan la dinámica económica de los centrales privados: El Estado se reservó importaciones adicionales, bien en forma de azúcar crudo o blanco; profundizó la regulación de precios a nivel de consumidor y negó licencias de importación en los períodos interzafras (julio- octubre). Sin embargo, en los últimos dos años, las estrategias desplegadas por los centrales azucareros privados, han posibilitado ciertos incrementos en la producción nacional. Este factor, combinado con una reducción en la tasa de crecimiento del consumo a causa de la recesión económica, ha disminuido el déficit de la producción nacional, que se estimó para el 2003 en 120.000 toneladas métricas de azúcar. Sin embargo, ese déficit se cubrió con importaciones de azúcar crudo por parte del sector privado y oficial.

El sector azucarero venezolano, requiere en este escenario, de ajustes inmediatos consistentes en: un nivel de precios que permita cubrir la estructura de costos de las empresas, se deben respetar las normativas de otorgamiento de licencias de importación, que el Estado no importe azúcares refinados y los venda a menor costo que el precio regulado y debe revisarse el esquema de exoneración de aranceles para el producto terminado.

\section{Perspectivas de la agroindustria azucarera venezolana}

La situación actual de la agroindustria azucarera en Venezuela, es francamente difícil. El precio de venta está estrictamente regulado por el Estado para proteger al consumidor, por lo que no se logran cubrir los costos de producción. Esto amenaza convertirse en la principal causa del derrumbe de la cañicultura nacional, y por tanto de la eficiencia agroindustrial. Se ha discutido mucho sobre el tema de los costos, llegándose a la conclusión de que todo el sector se podría encontrar muy cerca del umbral de cierre industrial de no mejorarse las condiciones del mercado. 
Si comparamos la situación de la agroindustria azucarera venezolana en 1981 con la situación actual, se puede apreciar que en aquel año el capital público representaba el $56 \%$ del sector (en un total de 20 empresas refinadoras), mientras que hoy en día esa participación es de apenas un 27\% (en un universo de 15 empresas). El proceso privatizador en la industria azucarera venezolana ha rendido frutos importantes, en cuanto a fortalecimiento de algunos determinantes de competitividad y manejo gerencial; mientras las empresas azucareras del Estado existentes en la actualidad representan un ejemplo de ineficiencia y pésimo manejo de las variables claves para un productor.

Las principales desventajas competitivas de la industria azucarera son las siguientes: altos niveles de inflación de costos de producción, caída del ingreso real del consumidor, altas tasas de interés para créditos de mediano plazo otorgados a los productores, elevados costos financieros de mantener inventarios, inestabilidad del signo monetario, carencia de políticas de apoyo al sector agrícola y agroindustrial, ausencia de un sistema aduanero confiable (debido a importaciones ilícitas de azúcares refinados), precios del azúcar regulados y desabastecimiento de materias primas. Estas desventajas han influido de manera negativa en los resultados obtenidos por el sector azucarero, contrayendo la actividad productiva, provocando excesiva capacidad instalada ociosa en los centrales, reduciendo la inversión por reducción de la relación costo- beneficio y generando una alarmante descapitalización de esta rama industrial. Sin embargo, en el período 1989-2003, que se ha presentado con características de la fase recesiva del ciclo económico, el sector ha ganado una mayor independencia con respecto a los subsidios y el apoyo del Estado.

Algunos sectores han planteado la necesidad de implementar planes para incrementar la capacidad industrial instalada mediante el aporte del financiamiento de la banca privada y de medidas de apoyo a los cañicultores en forma de sostenimiento de precios mínimos y subvenciones directas, a fin de incentivar la producción y productividad en la fase agrícola.

Por la situación antes expuesta, sería necesario solicitar al gobierno que se implemente un Programa de Medidas de Apoyo a la Cañicultura Nacional (no ligada a los precios), para incrementar la producción y la productividad en el cultivo y de esa forma influir en la reducción de la capacidad instalada ociosa de las empresas. Además, es necesario alertar al Ejecutivo Nacional sobre la situación de inseguridad en el campo, para que evite robos y secuestros. Asimismo, resulta estratégico implementar un mecanismo que permita la revisión periódica de los precios del azúcar de acuerdo a las fluctuaciones en los costos de producción, así como considerar el impacto de las políticas que estimulan la inflación y la depreciación del tipo de cambio.

Mediante la aplicación de las medidas correctivas señaladas, se alcanzaría el autoabastecimiento azucarero en el mercado nacional, con claras ventajas para el país, se eliminaría la dependencia de las importaciones, se generaría mayor valor agregado en el 
proceso productivo aprovechando de manera óptima los subproductos de la caña, para consolidar así una fundamental y amplia fuente de empleo en el país.

Tomando en cuenta la actual insuficiencia de la producción agrícola y la imperiosa necesidad de garantizar la "seguridad alimentaria" de la Nación, es indispensable poner en práctica políticas económicas claras y definidas con relación al impulso del sector agroindustrial, en general. En cuanto a la agroindustria azucarera, es necesario destacar su gran importancia no solamente como artículo de consumo, sino también como insumo para muchos y variados renglones industriales. Su recuperación y expansión podría contribuir a la generación de empleo productivo, que es en realidad la base de toda política social, y mejoramiento del nivel de vida de amplios sectores de la población, que están directa o indirectamente vinculados con dicha actividad.

Gráfico 1 - Producción de azúcar en Venezuela

(En toneladas métricas)

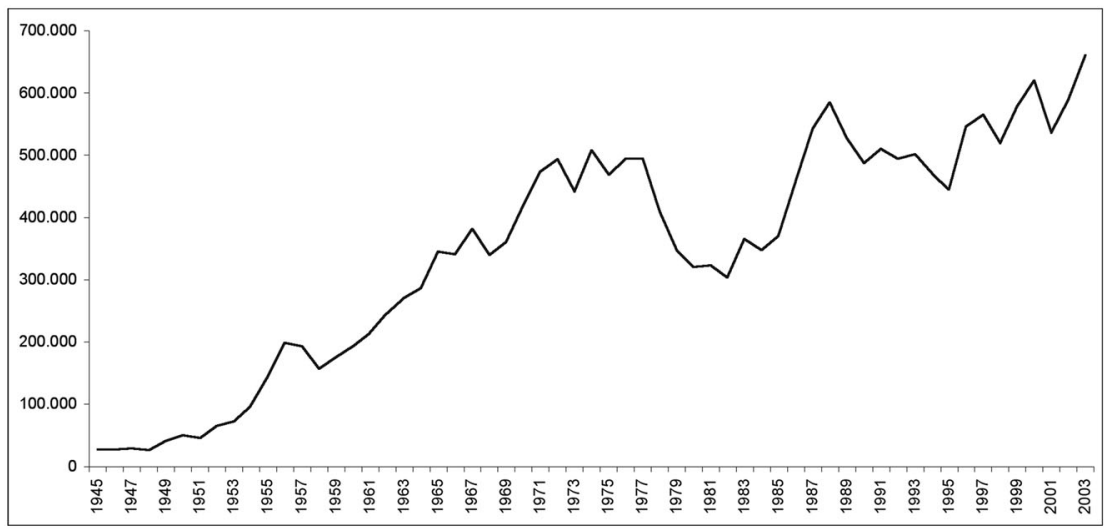

Fuente: Ministerios de Fomento, Agricultura y Cría y de Producción y Comercio, Memoria, años 1945-2003 


\section{Gráfico 2 - Producción y consumo nacional de azúcar}

(En toneladas métricas)

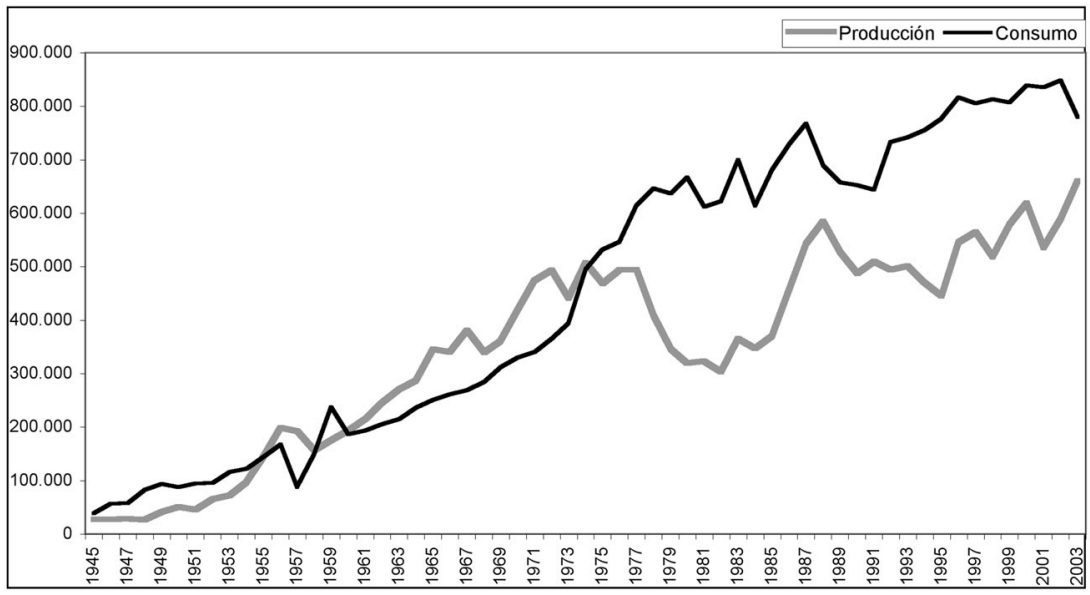

Fuente: Ministerios de Fomento, Agricultura y Cría y de Producción y Comercio,

Memoria, años 1945-2003

Gráfico 3 - Exportaciones e importaciones

(En toneladas métricas)

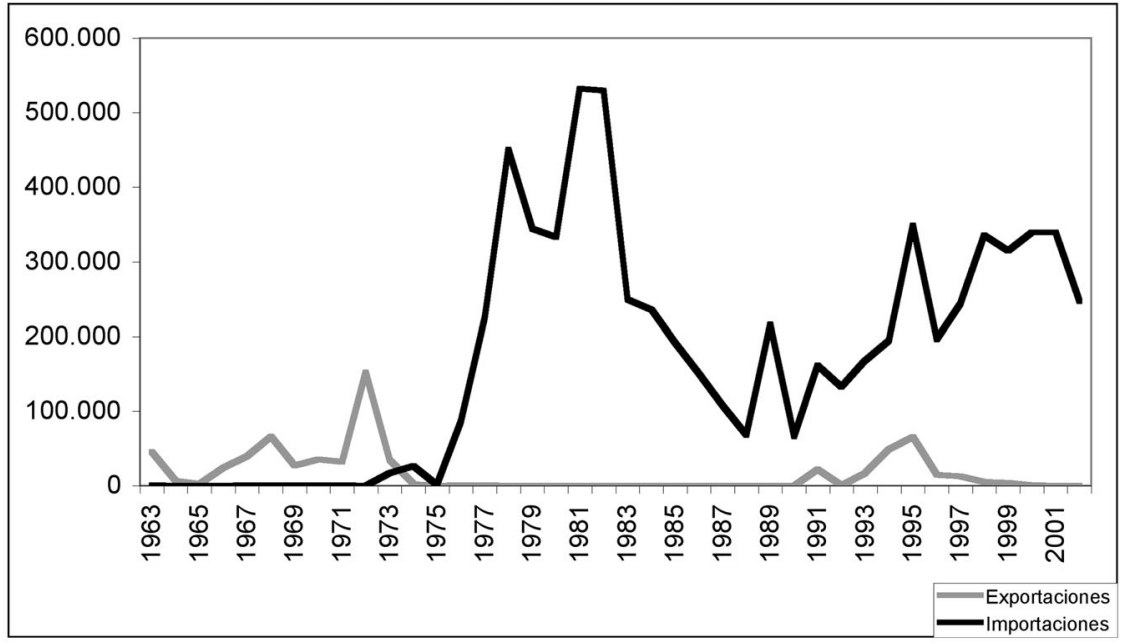

Fuente: Ministerios de Fomento, Agricultura y Cría y de Producción y Comercio, Memorias, años 1963-2001 


\section{Bibliografía}

ABARCA, Karelys; BANKO, Catalina. La politica económica y su influencia en el ciclo económico de la industria azucarera en el siglo XX. Ponencia presentada en Carora, II Congreso Azucarero Nacional, 2003.

ABARCA, Karelys; MAYORCA, E. Evolución de las formas de acumulación del capital en la industria venezolana durante la década de los noventa. Trabajo especial de grado. UCV, 2001.

ABREU, EDGAR, MARTÍNEZ, Z. et al. Entre campos y puertos...un siglo de transformaciones agroalimentarias en Venezuela. Caracas: Fundación Polar, 2001.

ATAVE. Diagnóstico de la situación actual de a industria azucarera nacional. Caracas: 1981.

BÁEZ, Mauricio. La situación relativa de la industria azucarera dentro de la economía venezolana. Caracas: DVA, 1981.

BANCO CENTRAL DE VENEZUELA. Series estadisticas de Venezuela en los últimos cincuenta años. Periodo 1940-1990. Caracas: BCV, 1991.

BANCO CENTRAL DE VENEZUELA. Anuario de estadísticas de precios y mercado laboral. Varios años.

BANKO, Catalina. El Central Venezuela y la industria azucarera zuliana 1913-1960. En: Akademos, Caracas, v. 5, 2003.

CENDES, Equipo de Economía Industrial. Procesos de industrialización periférica y formas de acumulación. La industria venezolana en los años setenta. Proyecto de Investigación. CENDES/CONICIT. Documento S1 - 1281, 1984.

CORDIPLAN. Planes de la Nación, n. IV, V, VIII, Plan 1970-1994.

CVF. Plan azucarero 1975 - 1980. Caracas: DVA, 1975.

DVA. El desarrollo de la industria azucarera en Venezuela y la necesidad de adoptar una nueva política de precios. Caracas: DVA, 1982.

EL ECONOMISTA. Ingenios centrales, Caracas, 19.06.1890.

FONDO DE INVERSIONES DE VENEZUELA. Proceso de privatización de las empresas públicas de la República de Venezuela. Caracas: FIV, 1994.

INSTITUTO DE COOPERACIÓNTÉCNICA INTERNACIONAL (IICA). Elmercado del azúcar en los países andinos. Lima, Perú: 2000.

MINISTERIO DE AGRICULTURA Y CRÍA. Anuario estadistico agropecuario. Varios años.

MINISTERIO DE AGRICULTURA Y CRÍA. Memorias. Varios años.

MINISTERIO DE FOMENTO. Memorias. Varios años.

MINISTERIO DE HACIENDA. Estadística Mercantily Marítima 1908-1930. Caracas, n. 309, julio. 1986 
OCEI. Encuestas industriales. Varios años.

PORTER, M. La ventaja competitiva de las naciones. Buenos Aires: J. Vergara Editorial, 1991.

RODRÍGUEZ, J. A. Paisajes geohistóricos cañeros en Venezuela. Caracas: Academia Nacional de la Historia, 1986.

TROCONIS, E. V. La tenencia de la tierra en el litoral central de Venezuela. Caracas: Editorial Equinoccio, 1979.

VENEZUELA AZUCARERA. Caracas, n. 8, junio. 1982.

VENEZUELA AZUCARERA. Caracas, Edición especial, marzo. 1997.

YÉPEZ, L. F. An evaluation of the Venezuelan sugar policy. Tesis PHD, University of Wisconsin, mimeo, 1970. 